\title{
Réflexions sur le respect des niveaux d'intervention dans un territoire contaminé par un dépôt accidentel de césium 137
}

\author{
H. MAUBERT*, D. ROBEAU*, P. RENAUD*, \\ I. LINGE**, O. PAVLOVSKI**, I. OSSIPIANTS**
}

(Manuscrit reçu le $1^{\text {er }}$ décembre 1994, révisé le 7 février 1995, accepté le 4 avril 1995)

RÉSUMÉ Après un accident nucléaire entraînant un dépôt important de radionucléides, les autorités responsables de la gestion des territoires contaminés sont confrontées à la question du respect des niveaux d'intervention. Ces niveaux sont exprimés de deux manières : soit sous forme d'activités massiques dans les produits alimentaires, soit sous forme de doses individuelles pour les populations. Un exercice de crise franco-russe a permis en 1993 de simuler une situation semblable pour un dépôt fictif de césium 137. Répondant aux questions de l'administration, les experts ont examiné à quelles conditions l'agriculture était possible dans le respect des niveaux d'intervention, selon d'abord le critère de concentration massique dans les produits alimentaires, puis selon une recommandation relative à une dose annuelle admissible pour les populations concernées de $1 \mathrm{mSv} / \mathrm{a}$, bien que cette limite ne soit actuellement applicable qu'aux situations normales et non post-accidentelles.

ABSTRACT After a nuclear accident leading to an important radionuclide deposition, the responsible authorities are faced to the question of complying with intervention levels. These regulations may be expressed as mass activities in foodstuffs, or individual doses for the populations. A situation of acute caesium 137 contamination of the environment was played in 1993 during a French and Russian joint crisis exercise. In answering the questions from the administration, the experts examined the conditions allowing agricultural practices 1) according to a set of intervention levels in foodstuffs, and 2) how that was compatible with a recommendation relative to an individual dose for the populations of $1 \mathrm{mSv} / \mathrm{y}$, although this limit is applicable in normal conditions and is not intended for post-accidental situations.

* Institut de protection et de sûreté nucléaire, CE Cadarache, 13108 Saint-Paul-lezDurance Cedex, France.

** Institut de Sûreté nucléaire, Bolshaya Tulskaya, 52, 113191 Moscou, Russie. 


\section{Introduction}

Un exercice franco-russe a eu lieu à Saint-Petersbourg du 21 au 25 juin 1993. Consacré aux problèmes de prise de décision pour la protection des populations et la réhabilitation des territoires en cas de contamination radioactive de zones étendues, il a été réalisé par l'Institut de sûreté nucléaire de l'Académie des sciences de Russie (IBRAE-RAN) et par l'Institut de protection et de sûreté nucléaire (IPSN) français avec la participation de l'administration de la région de Kalouga, territoire qui a été affecté par les retombées de l'accident de Tchernobyl.

Le scénario retenu était le suivant : dans la nuit du 16 au 17 mai 1993, un rejet de gaz et d'aérosols radioactifs provenant d'une centrale nucléaire fictive a eu lieu. Des dépôts radioactifs supérieurs à $555 \mathrm{kBq} / \mathrm{m}^{2}\left(15 \mathrm{Ci} / \mathrm{km}^{2}\right)^{1}$ en ${ }^{137} \mathrm{Cs}$ ont contaminé une superficie de $1500 \mathrm{~km}^{2}$. La radioactivité surfacique maximale atteint $3700 \mathrm{kBq} / \mathrm{m}^{2}\left(100 \mathrm{Ci} / \mathrm{km}^{2}\right)$. L'administration de Kalouga doit prendre des décisions visant à la protection des populations. Pour ce faire elle sollicite les conseils des experts de l'IBRAE et de l'IPSN et de diverses administrations russes. L'exercice se déroule en trois jours. Lors du premier jour on simule une situation correspondant au $16^{\mathrm{e}}$ jour après l'accident, le deuxième jour correspond à 1 an après, et le troisième jour à 5 ans après. Les experts se sont groupés par spécialité en équipes mixtes, russes et françaises.

Il est à remarquer que malgré la volonté de réaliser un exercice en prenant comme point de départ des hypothèses relatives à un événement fictif, la situation choisie ressemblait par bien des aspects à celle créée par l'accident de Tchernobyl. Les acteurs russes de l'exercice ont donc introduit dans les questions et dans les réponses de nombreux éléments empruntés aux situations qu'ils avaient vécues et étudiées.

Dans le présent document on traitera de certaines questions posées aux experts par l'administration, le $2^{\mathrm{e}}$ et le $3^{\mathrm{e}}$ jours. Ces questions, posées oralement, sont retranscrites en italiques au début des chapitres correspondants. Les évaluations concernent le seul césium 137 et ne peuvent pas toujours être généralisées à d'autres radionucléides tels que ${ }^{90} \mathrm{Sr}$ (émetteur $\beta$ pur) ou ${ }^{239} \mathrm{Pu}$ (émetteur $\alpha$ ).

\section{Les pratiques agricoles en territoires contaminés.}

Au début de la seconde journée de l'exercice, les experts savent que des mesures d'urgence ont été prises et que des régions ont été évacuées. Dans les zones où la population a été maintenue, l'administration désire savoir, un an après l'accident, à quelles conditions il est possible de pratiquer l'agriculture en fonction des diverses zones de dépôts. Faut-il réorienter la production agricole?

1. Dans toute la littérature russe, ukrainienne et bélarusse, l'unité de dépôt adoptée est le $\mathrm{Ci} / \mathrm{km}^{2}$. Ici l'unité est le $\mathrm{kBq} / \mathrm{m}^{2}$. Toutefois la valeur en $\mathrm{Ci} / \mathrm{km}^{2}$ est indiquée entre parenthèses : $37 \mathrm{kBq} / \mathrm{m}^{2}\left(1 \mathrm{Ci} / \mathrm{km}^{2}\right)$. 
Les évacuations ont été décidées en fonction des estimations de dose par irradiation externe pour l'année suivant l'accident. Le niveau d'intervention retenu était de $100 \mathrm{mSv}$, compte tenu des facteurs de protection dus aux habitations et aux mesures de décontamination envisagées. Sur le terrain, cela correspondait à des dépôts supérieurs à $1480 \mathrm{kBq} / \mathrm{m}^{2}\left(40 \mathrm{Ci} / \mathrm{km}^{2}\right)$ pour ${ }^{137} \mathrm{Cs}$. Un dépôt de ce seul radionucléide n'entraînerait pas une telle dose, mais il s'agit là d'un retour d'expérience de l'accident de Tchernobyl, où le dépôt était constitué d'un mélange de radionucléides.

\subsection{Relations entre valeurs de dépôts et activités massiques dans les produits agricoles}

Dans le temps limité dont les experts disposaient en situation d'exercice, seules des réponses génériques, prenant peu en compte la diversité pouvant exister au niveau local, pouvaient être fournies. Toutefois, chaque fois que possible, les experts se sont efforcés d'utiliser les données publiées relatives à la région de Kalouga ou de Briansk, de façon à proposer des réponses réalistes. Ces données concernent les facteurs de transfert dans la chaîne alimentaire et l'efficacité de contre-mesures éventuellement mises en œuvre.

Le critère retenu pour la décision de cultiver et de mettre sur le marché un produit est que sa radioactivité soit inférieure au niveau d'intervention russe dit NAT-94 (NAT : Niveaux admissibles temporaires), présenté sous forme de projet lors de l'exercice, c'est-à-dire sans valeur légale à ce moment là, mais toutefois très proche de la pratique réelle (Tab. I).

Il n'était pas possible dans le cadre de l'exercice de considérer l'ensemble de ces aliments. L'intérêt a été focalisé sur les 4 principales productions de la région de Kalouga, à savoir : les céréales représentant $39 \%$ des surfaces cultivées, les pommes de terre $(5 \%)$, le lait et la viande bovine. L'élevage bovin s'effectue sur $50 \%$ des surfaces cultivées. Sur le plan radioécologique ces produits peuvent être regroupés en 5 catégories :

- les céréales, notamment le blé, pour lequel on retiendra le niveau d'intervention relatif à la farine,

- les pommes de terre et autres légumes,

- le lait,

- le beurre,

- la viande bovine.

On considère que si, dans un système agricole donné, les niveaux d'intervention sont respectés pour ces produits, il n'y aura pas de difficultés majeures pour les autres tels que viande de porc et volailles (nourris hors sol), et les produits dérivés (conserves, jus,...). L'administration a décidé de plus de ne pas traiter le cas de l'eau potable, cet aliment ne posant généralement pas de problèmes urgents. 
TABLEAU I

Projet de niveau d'intervention en ${ }^{137} \mathrm{Cs}$ et ${ }^{134} \mathrm{Cs}$ dans les produits alimentaires et l'eau potable. Comparaison avec les niveaux européens [2]

Proposed intervention levels for ${ }^{134} \mathrm{Cs}$ and ${ }^{137} \mathrm{Cs}$ in foodstuffs and tapwater Comparison with european levels

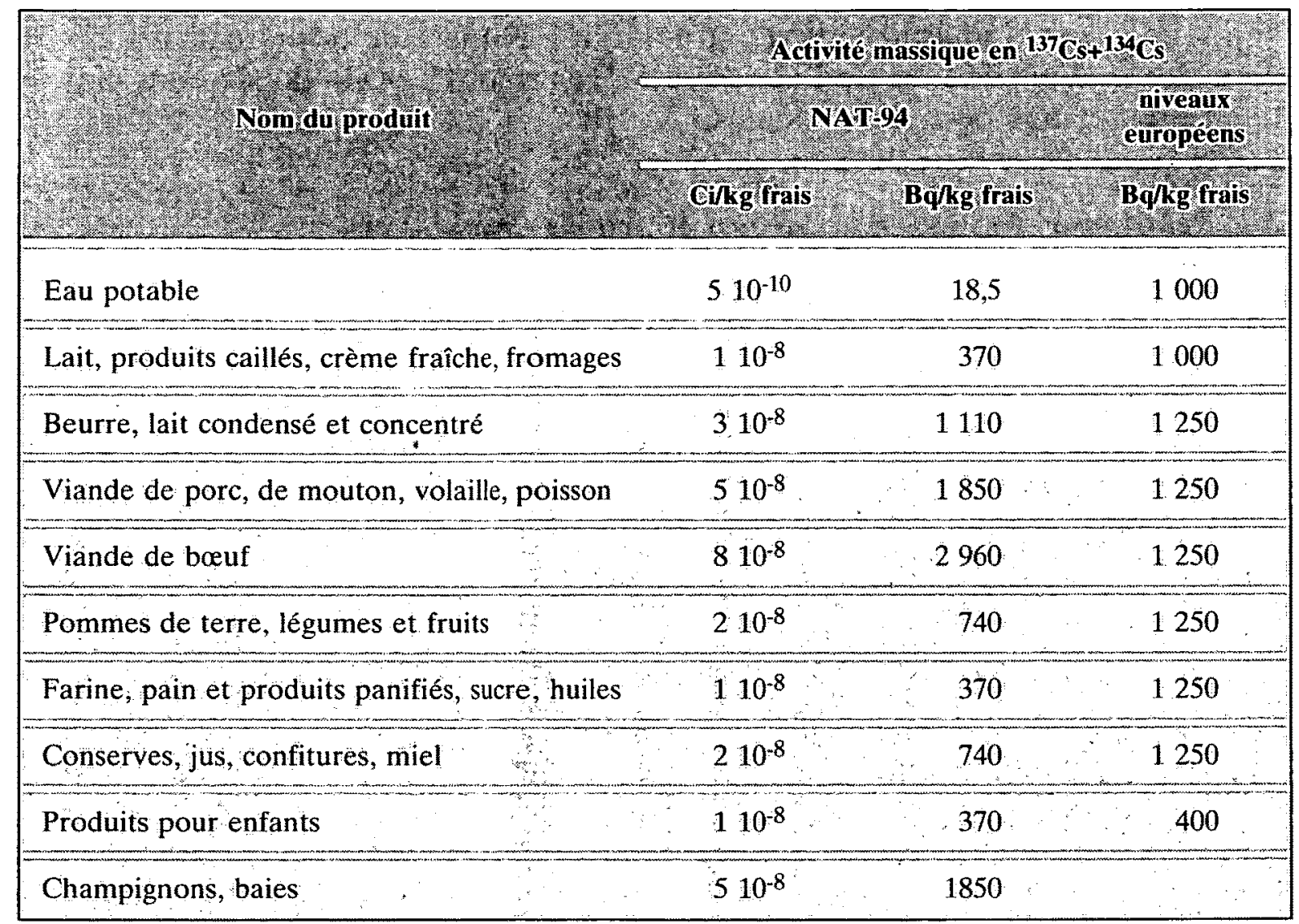

Pour chacune de ces productions, les seuils de dépôt en dessous desquels aucune précaution particulière n'est à prendre, les intervalles de dépôts où la mise en place de contre-mesures peut être efficace, et les niveaux au-dessus desquels telle ou telle culture doit être abandonnée vont être proposés.

Sur le terrain, il est notoire que les dépôts et les facteurs de transfert peuvent présenter une variabilité qui affecte largement la radioactivité des produits, parfois d'un facteur supérieur à celui caractérisant l'efficacité d'une contre-mesure (2 à 10). Rappelons qu'il s'agit ici d'un exercice et qu'il importait de tester modèles, raisonnement, calculs et communication entre les différents acteurs. Dans un cas réel de situation post-accidentelle, la diversité locale devrait être plus précisément appréhendée et pourrait l'être en raison du temps plus long dont les experts disposeraient.

\section{Les céréales}

La récolte dont il est question dans cette évaluation est celle ayant lieu un an après l'accident. Il n'y a donc aucun transfert direct de l'atmosphère à la 
plante. Les dépôts ont été homogénéisés par labourage dans la couche de sol arable puis le semis a eu lieu. La contamination s'effectue par transfert racinaire. Pour les années suivant l'accident de Tchernobyl, les auteurs russes ont publié des valeurs de facteurs de transfert exprimées en activités massiques observées dans la partie consommable des plantes, ici le grain, rapportées au dépôt, selon les types de sols et les pratiques agricoles. Dans le cas de pratiques agricoles normales, Alexakhin [1] propose des valeurs variant de 0,2 à $0,4 \mathrm{~Bq} / \mathrm{kg} / \mathrm{kBq} / \mathrm{m}^{2}$ pour les céréales cultivées sur un sol sableux, de 0,1 à 0,2 pour un sol limoneux et de 0,04 à 0,15 pour un sol argileux. Si des contremesures telles que l'ajout d'engrais sont appliquées, ces valeurs varient respectivement de 0,15 à 0,20 (sol sableux), 0,07 à 0,08 (sol limoneux) et 0,03 à 0,06 (sol argileux). Nous retiendrons la valeur maximale de $0,4 \mathrm{~Bq} / \mathrm{kg} / \mathrm{kBq} / \mathrm{m}^{2}$ relative au blé d'hiver sur des sols sableux, largement répandus dans la région considérée. On en déduit que si l'on retient une valeur de $370 \mathrm{~Bq} / \mathrm{kg}$ dans le grain, le dépôt correspondant est de $925 \mathrm{kBq} / \mathrm{m}^{2}\left(25 \mathrm{Ci} / \mathrm{km}^{2}\right)$. En dessous de cette valeur on peut donc partout cultiver les céréales. Si une contre-mesure telle que l'ajout d'engrais potassique est utilisée, cette valeur peut être multipliée par 2.

\section{Les pommes de terre et autres légumes}

Le raisonnement est similaire à celui adopté pour les céréales. Le facteur de transfert maximal mentionné par Alexakhin est de $0,2 \mathrm{~Bq} / \mathrm{kg} / \mathrm{kBq} / \mathrm{m}^{2}$. Le niveau d'intervention adopté étant de $740 \mathrm{~Bq} / \mathrm{kg}$, on trouve que le dépôt maximal admissible pour ce type de cultures est de $3700 \mathrm{kBq} / \mathrm{m}^{2}\left(100 \mathrm{Ci} / \mathrm{km}^{2}\right)$. Des contre-mesures du type ajout d'engrais permettraient de gagner un facteur compris entre 2 et 3 .

\section{Le lait}

Si l'on prend une quantité ingérée par la vache de $13 \mathrm{~kg}$ de matière sèche par jour et un facteur de transfert à l'équilibre entre la nourriture et le lait de $810^{-3} \mathrm{~Bq} / \mathrm{kg} / \mathrm{Bq} / \mathrm{j}$, [3] sachant que le niveau d'intervention est de $370 \mathrm{~Bq} / \mathrm{l}$, la radioactivité de l'herbe ne doit pas dépasser $3560 \mathrm{~Bq} / \mathrm{kg} \mathrm{sec}$.

Dans les pratiques agricoles actuellement courantes en Russie, les vaches pâturent sur deux types de prairie: d'une part les prairies permanentes naturelles, qui n'ont pas subi de traitement particulier, d'autre part les prairies semées sur des terrains qui ont été labourés et améliorés par l'adjonction d'engrais.

Dans le premier cas, le facteur de transfert global à l'herbe est d'environ $20 \mathrm{~Bq} / \mathrm{kg} \mathrm{sec} / \mathrm{kBq} / \mathrm{m}^{2}$ [1]. Le dépôt maximal associé au niveau d'intervention est donc de $178 \mathrm{kBq} / \mathrm{m}^{2}\left(5 \mathrm{Ci} / \mathrm{km}^{2}\right)$. L'adjonction de complexants (hexacyanoferrate (AFCF), dérivés du bleu de Prusse) à la ration alimentaire abaisse le transfert métabolique au lait d'un facteur 5 à 10 [9]. Retenons le chiffre 5. Cette contre-mesure permet donc de laisser paître les vaches sur des prairies naturelles où les dépôts sont inférieurs à $925 \mathrm{kBq} / \mathrm{m}^{2}\left(25 \mathrm{Ci} / \mathrm{km}^{2}\right)$. 
Dans le second cas, le labourage et l'amendement du sol des prairies permettent de réduire la radioactivité de l'herbe d'un facteur de 3 à 15 selon les sols [5]. En prenant la valeur. 3, relative aux sols les moins bons, on en déduit qu'en l'absence de toute autre contre-mesure les vaches peuvent pâturer sur des zones où le dépôt est inférieur à $555 \mathrm{kBq} / \mathrm{m}^{2}\left(15 \mathrm{Ci} / \mathrm{km}^{2}\right)$, et que cette limite peut théoriquement être multipliée par 5 par l'administration d'hexacyanoferrate aux animaux.

\section{Le beurre}

Le beurre présente une radioactivité spécifique en ${ }^{137} \mathrm{Cs} 100$ fois inférieure à celle du lait dont il est issu [6]. Le niveau d'intervention pour le beurre est de $1110 \mathrm{~Bq} / \mathrm{kg}$, c'est-à-dire trois fois plus élevé que pour le lait. Donc, pour trouver les valeurs de dépôts associées aux niveaux d'intervention dans le beurre, il est théoriquement possible de reprendre celles relatives au lait et les multiplier par 300 .

\section{La viande bovine}

Comme pour le lait, on peut distinguer les prairies naturelles et les prairies cultivées. Le facteur de transfert retenu à l'équilibre entre la nourriture et la viande est de $510^{-2} \mathrm{j} / \mathrm{kg}$ [3]. Le niveau d'intervention relatif à la viande bovine est de $2960 \mathrm{~Bq} / \mathrm{kg}$. Par un calcul similaire à celui réalisé pour le lait, on trouve que, sans contre-mesure, la pâture sur prairie naturelle est possible jusqu'à des dépôts de $228 \mathrm{kBq} / \mathrm{m}^{2}\left(6 \mathrm{Ci} / \mathrm{km}^{2}\right)$. L'adjonction d'hexacyanoferrate à la ration alimentaire permet d'envisager la production de viande jusqu'à des dépôts de $1140 \mathrm{kBq} / \mathrm{m}^{2}\left(30 \mathrm{Ci} / \mathrm{km}^{2}\right)$. Sur prairie cultivée, sans contre-mesure, le seuil est de $666 \mathrm{kBq} / \mathrm{m}^{2}\left(18 \mathrm{Ci} / \mathrm{km}^{2}\right)$, et avec $\mathrm{AFCF}$ il est égal à $3330 \mathrm{kBq} / \mathrm{m}^{2}$ $\left(90 \mathrm{Ci} / \mathrm{km}^{2}\right)$.

Une contre-mesure efficace et largement utilisée pour réduire la contamination de la viande consiste à trier les animaux par une mesure in vivo au moment de l'abattage, et à faire subir aux bêtes dont la radioactivité est jugée trop élevée, une cure de décontamination avec des nourritures non radioactives. La période effective de décontamination est de $30 \mathrm{j}$ pour le césium [4].

\subsection{Récapitulation et conclusion sur les pratiques agricoles}

Les seuils précédemment calculés sont récapitulés dans la figure 1. On constate que les productions qui peuvent poser le plus de problèmes sont d'abord le lait puis la viande bovine. Si l'abattage différé peut autoriser la récupération d'un animal trop contaminé, il n'en est pas de même pour la production de lait, qui est continue. Dans ce cas, l'utilisation d'une contre-mesure comme l'administration d'hexacyanoferrate peut être de la plus grande utilité pour respecter les niveaux d'intervention et réduire les doses individuelles et collectives. D'ailleurs cette contre-mesure semble être la seule largement utilisée en Russie, en Ukraine et en Bélarus. La transformation en beurre permet de rentabiliser les stocks de lait qui malgré l'utilisation de contre-mesures ne 
seraient pas consommables. Cela est effectivement pratiqué à grande échelle ; dans certaines régions contaminées, la production de lait est destinée à la transformation en beurre, et le lait de consommation est importé. En raison du manque de temps, les contre-mesures ayant trait au remplacement des cultures, c'est-à-dire à la réorientation du système agro-industriel, n'ont pas été considérées.

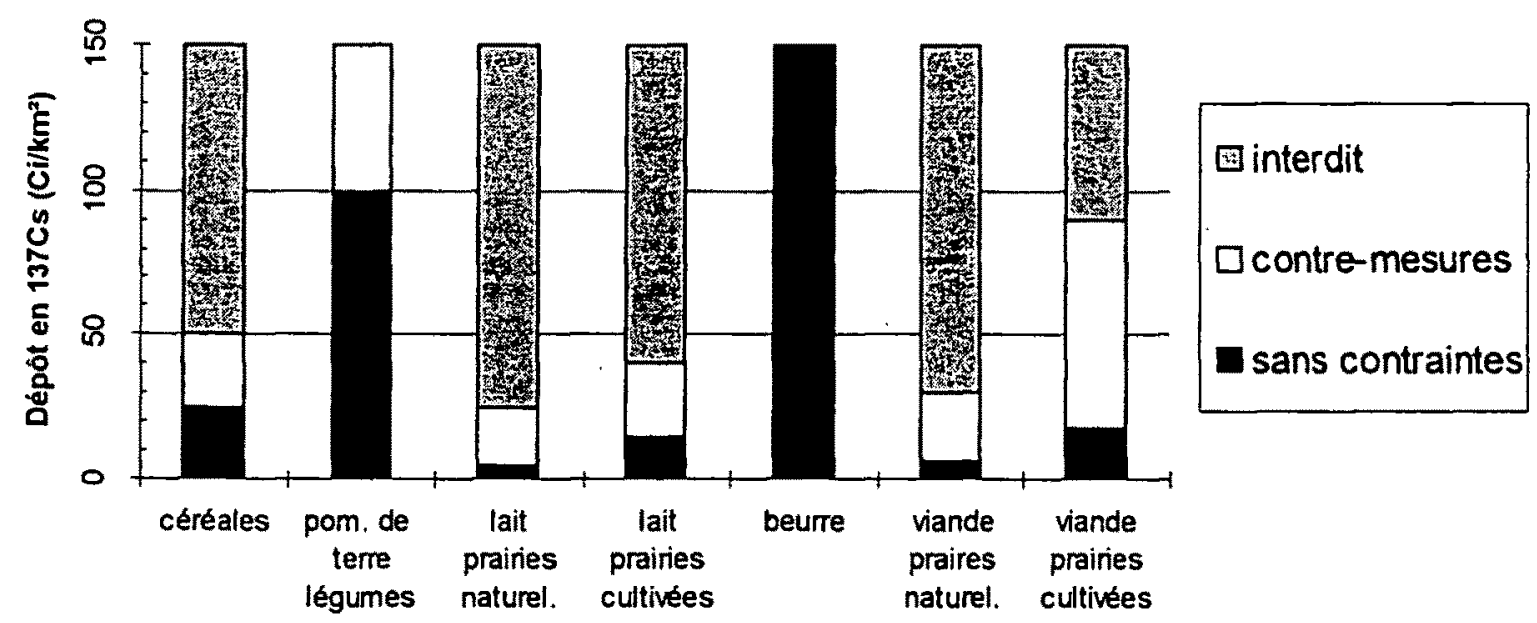

Fig. 1 - Dépôts au sol maximaux admissibles pour atteindre les niveaux d'intervention relatifs aux produits agricoles (base ${ }^{13} \mathrm{Cs}$ ).

Maximum permissible soil deposits in order to reach the intervention levels for foodstuffs (based on ${ }^{137}$ Cs).

Tous les calculs précédents sont relatifs à la première année qui suit un dépôt de ${ }^{137} \mathrm{Cs}$. Peu à peu, à la suite de la migration du radionucléide et de son immobilisation croissante dans les matrices minérales des sols, les facteurs de transfert décroissent. Selon des données communiquées aux experts durant l'exercice, on estime que cinq ans après l'accident, il faut multiplier les facteurs de transfert relatifs à la première année par 0,65 environ ${ }^{1}$. Cela permet de relever dans des proportions semblables les dépôts indiqués.

\section{Possibilités de minimisation de l'exposition des populations et retour à la normale}

Lors de la troisième journée de l'exercice, pendant laquelle était jouée la situation qui prévaudrait cinq ans après l'accident, une des questions posées aux experts concernait le retour à des conditions qualifiées de normales dans les zones contaminées.

1. Cette valeur n'a pas été justifiée ; c'est un retour d'expérience de Tchernobyl. Un calcul réalisé avec le modèle ECOSYS-87 [8] donne un facteur de réduction de 0,72 en 4 ans. 
Les représentants de l'administration souhaitaient savoir dans quelle mesure il était possible de respecter pour le public une dose de $1 \mathrm{mSv} / \mathrm{a}$, sur des territoires contaminés par des dépôts compris entre 370 et $1480 \mathrm{kBq} / \mathrm{m}^{2}$ (1 et $40 \mathrm{Ci} / \mathrm{km}^{2}$ ) pour le césium 137. Au cas où le respect de cette dose serait impossible, il était demandé aux experts de préciser quelle dose minimale pouvait être raisonnablement attendue.

Lors de la préparation et de l'exécution de l'exercice, l'IBRAE a fourni aux intervenants des bases de données portant sur la situation radioactive dans les localités concernées de la région de Kalouga. Ces bases contenaient :

- des informations géographiques et démographiques,

- les doses sur l'année dues à l'irradiation $\gamma$,

- les doses engagées sur l'année dues à l'ingestion,

- les dépôts minimaux, moyens et maximaux par localité,

- d'autres informations qui ne seront pas utilisées ici, comme le nombre de personnes évacuées, les mesures de contamination des denrées alimentaires, ...

Ces bases de données sont dérivées de celles établies après l'accident de Tchernobyl dans la région de Briansk. Les niveaux de contamination relatifs à telle ou telle localité ont pu être modifiés ou transposés pour les besoins de l'exercice. Les bases sont structurées par localités (fermes, villages, bourgs). Pour chaque localité, il y a au moins une mesure de dépôt, souvent complétée par des mesures dans les produits agricoles, des mesures de débit de dose à l'intérieur et à l'extérieur des maisons,... Les valeurs manquantes sont reconstituées par des modèles empiriques résultant des observations effectuées dans des conditions comparables (dans la localité voisine, par exemple). Les hypothèses concernant la ration alimentaire correspondent à des moyennes observées. Les résultats des calculs d'incorporation sont contrôlés par anthropogammamétrie sur de nombreuses personnes appartenant à des groupes socioprofessionnels représentatifs. Malheureusement l'ensemble des travaux ayant servi à établir ces bases ne nous a pas été communiqué. Toutefois, pour les besoins de l'exercice, en fonction des dépôts indiqués, les informations telles que débits de dose ou autres restent réalistes, de sorte qu'il est possible de bâtir un raisonnement de portée assez générale.

\subsection{Conséquences d'un niveau d'intervention de $1 \mathrm{mSv} / \mathrm{a}$}

La question posée est complexe dans la mesure où toutes les voies d'atteinte, ingestion, irradiation externe en milieu rural ou urbain, etc., sont concernées. Il faut donc faire la part de chacune des voies d'atteinte et étudier dans quelle mesure il est possible d'entreprendre des actions visant, si nécessaire, à réduire les doses engagées.

Le tableau AI (annexe) et la figure 2 fournissent par grande zone de dépôt, les doses par irradiation externe et par ingestion pour l'année courante (1998, soit 5 ans après l'accident supposé). Les valeurs mentionnées sous la rubrique 
"irradiation externe" sont évaluées en prenant en compte les facteurs de protection dus aux habitations, les habitudes professionnelles et les performances des mesures de décontamination. Un autre champ de la base de données contient ces mêmes estimations effectuées sans tenir compte de facteurs de protection, ce qui est peu réaliste et n'a pas été utilisé dans la présente étude. Les valeurs représentées ci-après sont des moyennes arithmétiques. Nous avons vérifié que pour un intervalle de dépôt, la répartition des valeurs indiquées dans la base de données suivait raisonnablement une loi normale.

On constate à la lecture de la figure 2 que le niveau de $1 \mathrm{mSv} / \mathrm{a}$ ne paraît accessible que pour des dépôts inférieurs à $185 \mathrm{kBq} / \mathrm{m}^{2}\left(5 \mathrm{Ci} / \mathrm{km}^{2}\right)$. Encore faudrait-il faire un léger effort de décontamination dans les zones où les dépôts sont proches de cette valeur.

\section{Analyse des valeurs pour la voie "irradiation externe"}

Dès que le dépôt excède $185 \mathrm{kBq} / \mathrm{m}^{2}\left(5 \mathrm{Ci} / \mathrm{km}^{2}\right)$, la dose par irradiation externe dépasse $1 \mathrm{mSv} / \mathrm{a}$. Pour respecter cette valeur, ou du moins s'en rapprocher, il conviendrait de réduire l'irradiation par des mesures de décontamination. Citons en particulier:

- le changement du toit des maisons,

- le décapage ou le labourage des jardins qui les entourent, notamment de la zone située au droit des gouttières,

- le décapage des surfaces urbaines telles que routes, trottoirs...

- éventuellement, le recouvrement de surfaces contaminées par un matériau qui fait écran, et qui emprisonne la radioactivité,

- le labourage des champs et surfaces toujours en herbe,

- les restrictions des activités récréatives en forêt.

Pour l'irradiation externe, le labourage est crédité d'une efficacité allant de $50 \%$ à $85 \%$ [6-7] et le décapage de 80 à $95 \%$ [7].

Toutefois, la littérature fournit peu de valeurs précises quant à l'efficacité1 réelle, le coût et les modalités de mise en œuvre à grande échelle d'un jeu de ces contre-mesures.

\section{Analyse des valeurs pour la voie "ingestion"}

Pour l'ingestion la valeur de $1 \mathrm{mSv} / \mathrm{a}$ n'est atteinte qu'à partir de dépôts supérieurs à $555 \mathrm{kBq} / \mathrm{m}^{2}\left(15 \mathrm{Ci} / \mathrm{km}^{2}\right)$. Entre 555 et $1480 \mathrm{kBq} / \mathrm{m}^{2}$ (15 et $40 \mathrm{Ci} / \mathrm{km}^{2}$ ), l'infléchissement de la courbe et la stabilisation des doses à des valeurs inférieures à $1,2 \mathrm{mSv} / \mathrm{a}$ traduisent l'effort fait par les autorités pour assurer un approvisionnement en denrées peu contaminées. Il semble, toute-

1. Si $A$ est la dose en l'absence d'une contre-mesure, et $B$ la dose après application de cette contre-mesure, on entend par efficacité le rapport $(A-B) / A$, exprimé en pourcentage. 
fois, que cet effort a atteint ses limites, du moins dans les régions concernées ou règnent de façon endémique une certaine pénurie alimentaire et des problèmes de transport.

\section{Addition des deux voies et proposition}

Supposons que l'effort fait pour fournir des denrées alimentaires non contaminées ne puisse être augmenté et qu'une décontamination supplémentaire des lieux d'activité soit efficace à $50 \%$, cette dernière valeur étant avancée de façon arbitraire. En réponse à l'administration, une relation entre les doses raisonnablement attendues et les valeurs du dépôt est mentionnée dans le tableau II.

Toutes ces estimations sont relatives à la situation prévalant 5 ans après l'accident. Compte tenu de la période de décroissance radioactive du césium 137,30 ans, et de sa forte fixation dans les sols, la situation ne peut s'améliorer que lentement.

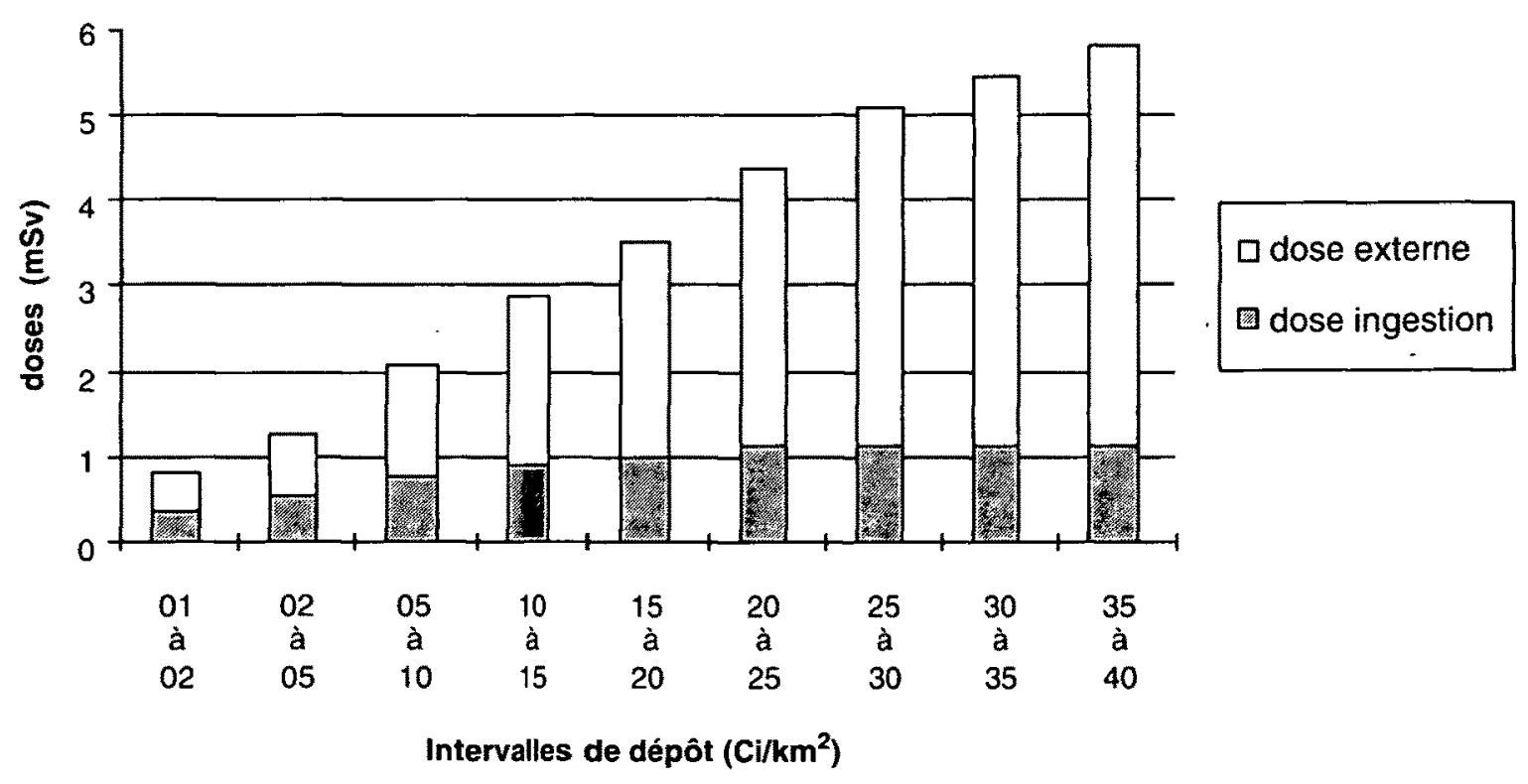

Fig. 2 - Distribution des doses pour la cinquième année après l'accident.

Dose distribution on the $5^{\text {th }}$ year following the accident.

\subsection{Discussion sur la cohérence des niveaux d'intervention relatifs aux doses délivrées aux populations et des niveaux d'intervention sur les produits alimentaires}

A ce stade des réflexions sur les conséquences d'un rejet important de radiocésium, on peut envisager d'aborder la question du maintien de la pratique agricole dans les territoires non évacués. 
TABLEAU II

Doses attendues pour la $5^{\mathbf{e}}$ année après l'accident; valeurs établies avec les hypothèses de l'exercice de crise IPSN/IBRAE Expected doses 5 years after the accident;

values established with the assumptions of the IPSN/IBRAE crisis exercise

\begin{tabular}{|c|c|c|}
\hline & $\begin{array}{c}\text { Intervalle de dépót } \\
\left(\mathbf{C i} / \mathbf{k m}^{2}\right)\end{array}$ & $\begin{array}{c}\text { Dose attendues } \\
\left(\mathbf{k B q} / \mathbf{m}^{2}\right)\end{array}$ \\
\hline $0-5$ & $0-185$ & 1 \\
\hline $5-15$ & $185-555$ & 2 \\
\hline $15-25$ & $555-925$ & 3 \\
\hline $25-40$ & $925-480$ & 4 \\
\hline
\end{tabular}

Les résultats mentionnés en 2 ont été établis en tenant compte des propositions de niveaux d'intervention russes, mais on peut réaliser une transposition au cas européen en utilisant ceux publiés dans le Journal officiel des communautés européennes [2]. Dans le tableau III, sont mentionnés par catégorie de produits alimentaires, les dépôts associés aux divers niveaux d'intervention. On a tenu compte de l'atténuation des facteurs de transfert entre la $1^{\text {re }}$ et la $5^{\mathrm{e}}$ année après l'accident.

Selon les niveaux d'intervention russes, la production pour laquelle on atteint le plus rapidement une interdiction de consommation est celle du lait sur pâture naturelle. C'est d'ailleurs, de fait, la voie d'atteinte prépondérante par ingestion. Selon les niveaux du Journal officiel des Communautés européennes [2], la production de viande bovine sur prairie naturelle est le premier problème rencontré. Toutefois, l'utilisation de compléments alimentaires non contaminés, et l'usage de cures de décontamination pourraient facilement venir à bout de cette difficulté.

Pour le reste on constate que les dépôts limites sont en général supérieurs à $370 \mathrm{kBq} / \mathrm{m}^{2}\left(10 \mathrm{Ci} / \mathrm{km}^{2}\right)$, parfois $3700 \mathrm{kBq} / \mathrm{m}^{2}\left(100 \mathrm{Ci} / \mathrm{km}^{2}\right)$, et ce malgré la prise en compte, pour l'ensemble des estimations, des mêmes facteurs de transferts relatifs aux sols russes choisis parmi les plus défavorables. En France, par exemple, les sols, de meilleure qualité, conduisent à des facteurs de transfert significativement plus faibles.

Nous avons vu, par ailleurs, que la seule irradiation externe conduisait à une dose de $1 \mathrm{mSv} / \mathrm{a}$ pour un dépôt supérieur à $185 \mathrm{kBq} / \mathrm{m}^{2}\left(5 \mathrm{Ci} / \mathrm{km}^{2}\right)$. On en déduit que si un tel critère doit être adopté, ce sera le facteur limitant. En d'autres termes, si la dose par irradiation externe délivrée aux cultivateurs est inférieure à $1 \mathrm{mSv} / \mathrm{a}$, alors leur production agricole présentera certainement 
TABLEAUं III

Limites de commercialisation des produits agricoles, et dépôts maximaux admissibles sur la base du ${ }^{137} \mathrm{Cs}$ Marketing limits for foodstuffs and maximum permissible deposits (based on ${ }^{137} \mathrm{Cs}$ )

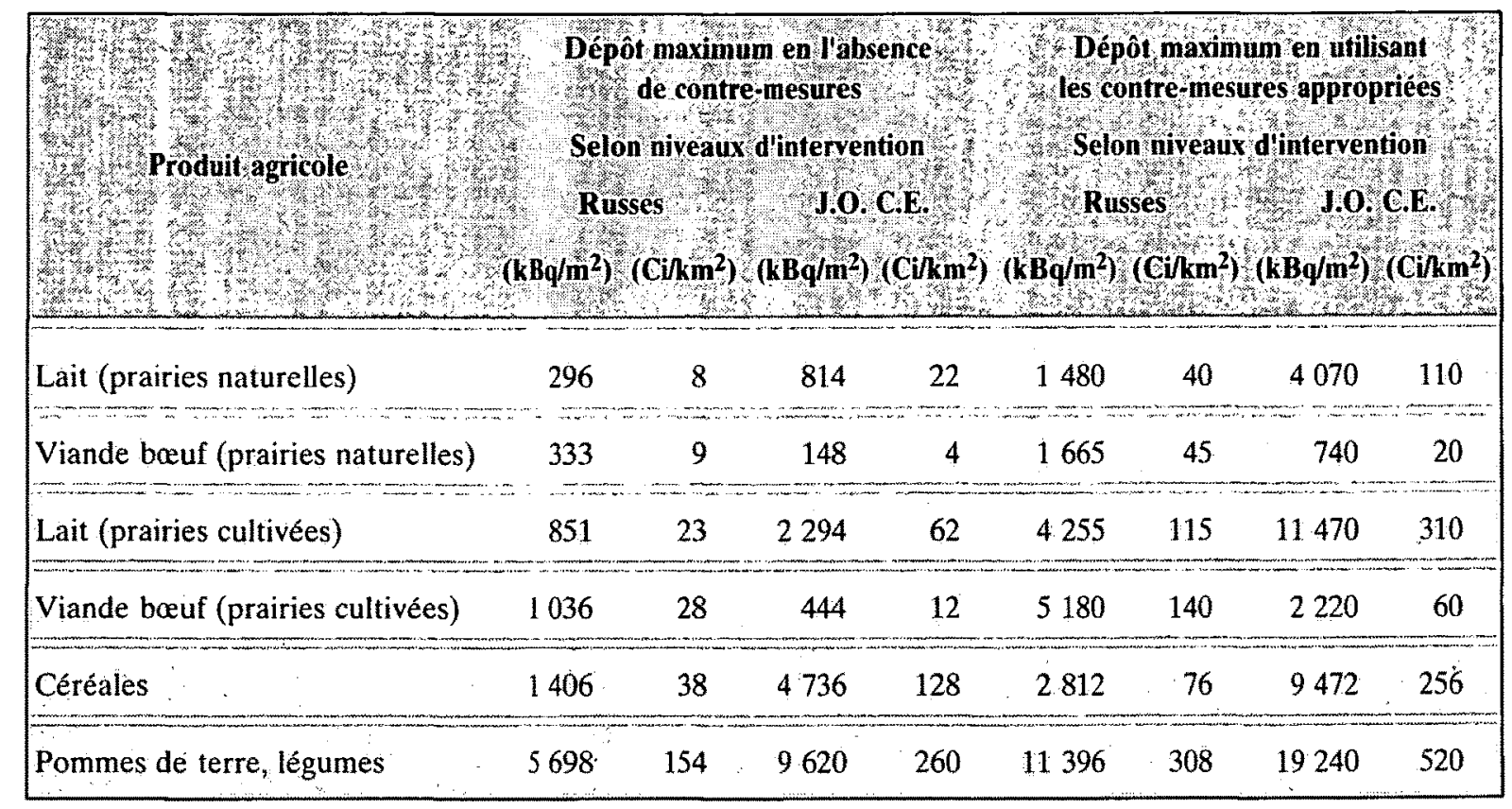

une radioactivité inférieure aux niveaux d'intervention, en dehors peut-être de la viande bovine pour laquelle des contre-mesures simples peuvent être prises. Cette conclusion, établie sur la base d'un dépôt de césium 137, peut sans risque être généralisée à d'autres radionucléides émetteurs $\gamma$ de forte énergie, tel que ${ }^{60} \mathrm{Co}$ qui, en exposition externe, est 5 fois plus pénalisant que ${ }^{137} \mathrm{Cs}$ et pour lequel les facteurs de transfert aux plantes sont comparables ou inférieurs. Cela ne serait pas vrai pour un émetteur $\beta$ pur comme ${ }^{90} \mathrm{Sr}$, ou un émetteur $\alpha$ comme ${ }^{239} \mathrm{Pu}$.

De plus, dans les zones où l'irradiation externe seule entraînerait une dose supérieure à $1 \mathrm{mSv} / \mathrm{a}$, la consommation de produits locaux, bien que présentant une activité inférieure aux niveaux d'intervention, pourrait se trouver interdite. Cette même production pourrait légalement être exportée. Les populations résidant dans la zone contaminée se nourriraient alors de produits provenant d'endroits exempts de contamination. Il s'agit bien entendu là d'une hypothèse académique. On peut s'interroger sur la validité d'un tel schéma au regard de l'opinion publique.

Si le niveau d'intervention en matière de dose était relevé, ce qui est envisageable en situation post-accidentelle, on rencontrerait progressivement des problèmes d'activité dans les aliments, en commençant par les produits d'origine animale, viande et lait. C'est là que pourrait se déployer une palette de contre-mesures de façon à réduire aussi bas que raisonnablement possible l'exposition des populations, compte-tenu des facteurs économiques et sociaux. 


\section{Conclusion}

La préparation de l'exercice de prise de décisions de Saint Petersbourg et sa réalisation ont permis aux experts de se confronter aux questions concrètes posées par l'administration. Cela a induit une réflexion globale sur l'utilisation des valeurs relatives aux transferts des radionucléides. Toutefois il convient de s'interroger sur les limites d'un tel exercice. En particulier :

- l'efficacité des contre-mesures n'a été analysée que d'un point de vue radiologique, pour des doses individuelles, et une étude d'optimisation faisant intervenir d'autres critères (de doses collectives, économiques, sociaux, écologiques, etc.) serait nécessaire pour mieux définir les seuils d'intervention, et optimiser la gestion des territoires contaminés ;

- les problèmes posés par le milieu urbain n'ont pas été traités ; c'est pourtant en ville que vivent la plupart des gens;

- la prise en compte du volume éventuel de déchets générés selon les compartiments de l'environnement et leur gestion n'ont pas été abordées.

Les conclusions pratiques tirées de cet exercice concernent la nécessité d'intensifier nos efforts en ce qui concerne le milieu urbain, et de disposer d'un outil informatique capable de prendre en compte la multiplicité des voies de transfert possibles, ainsi que la diversité des situations locales.

Il conviendrait donc, dans l'avenir, d'effectuer plus d'études ayant un caractère global, multiparamétrique. Quelles sont les voies d'atteintes des populations les plus contraignantes dans l'absolu, ou vis-à-vis de telle ou telle réglementation ou recommandation, quelle est l'évolution à long terme des phénomènes, à quelle échelle de temps, de quelle manière vont intervenir les éléments socio-économiques, etc. ?

\section{RÉFÉRENCES}

[1] ALEXAKHIN R.M. - General principles applied in the use of countermeasures. Sci. Total Environ., 1993, 137, 9-20.

[2] COMMUNAUTÉS EUROPÉENNES. Conseil. Niveaux maximaux admissibles pour les denrées alimentaires et les aliments pour bétail. Règlement EURATOM 2218-89 du 18 juillet 1989, J.O.C.E., 22 juillet 1989, L 211, 1-3.

[3] COUGHREY P.J. Radioactivity transfer to animal products. Rapport EUR 12603 EN, 1991 , p. 17.

[4] ILIAZOV R.G., FIRSAKOVA S.K., KARPENKO A.F. - Problèmes radioécologiques de l'élevage en Biélorussie après l'accident de Tchernobyl. In: Résumé détaillé des communications présentées au séminaire "Radioécologie et contre-mesures", Kiev, mai 1991. Saval : Union internationale des radioécologistes, 1991, p. 82.

[5] ILliNe M.I., PEREPELIATNIKOV G.P., PRISTER S. - Amélioration radicale des prairies contaminées : meilleur moyen de diminution de ${ }^{137} \mathrm{Cs}$ dans 1 'herbe. In : Résumé détaillé des communications présentées au séminaire "Radioécologie et contre-mesures", Kiev, mai 1991. Saval: Union internationale des radioécologistes, 1991, p. 66. 
[6] McENRI C.M., MITCHELL P.I., CUNNINGHAM J.D. - The transfer of radiocaesium from whole milk to milk products. In: Seminar on Radioactivity transfer during food processing and culinary preparation, Cadarache, Septembre 1989. Luxembourg: CCE DG XI, 1989, 251-273.

[7] MAUBERT H., VOVK I., ROED J., ARAPIS G., JOUVE A. - Reduction of soilplant transfer factor; mechanical aspects. Sci. Total Environ., 1993, 137, 163-167.

[8] MÜLLER H., PRÖHL G. - ECOSYS-87 : A dynamic model for assessing radiological consequences of nuclear accidents. Health Phys., 1993, 64(3), 232-252.

[9] VOIGT G. - Chemical methods to reduce the radioactive contamination of animals and their products in agricultural ecosystems. Sci. Total Environ., 1993, 137, 205-225.

\section{Annexe}

\section{TABLEAU A1}

Doses prévues, par voie d'atteinte et par zone de dépôt, à 5 ans et sur la période 6 à 70 ans après l'accident supposé (extrait des bases IBRAE) Expected doses 5 y and from 6 to 70 y after the supposed accident according to the pathways and deposit areas (after IBRAE data)

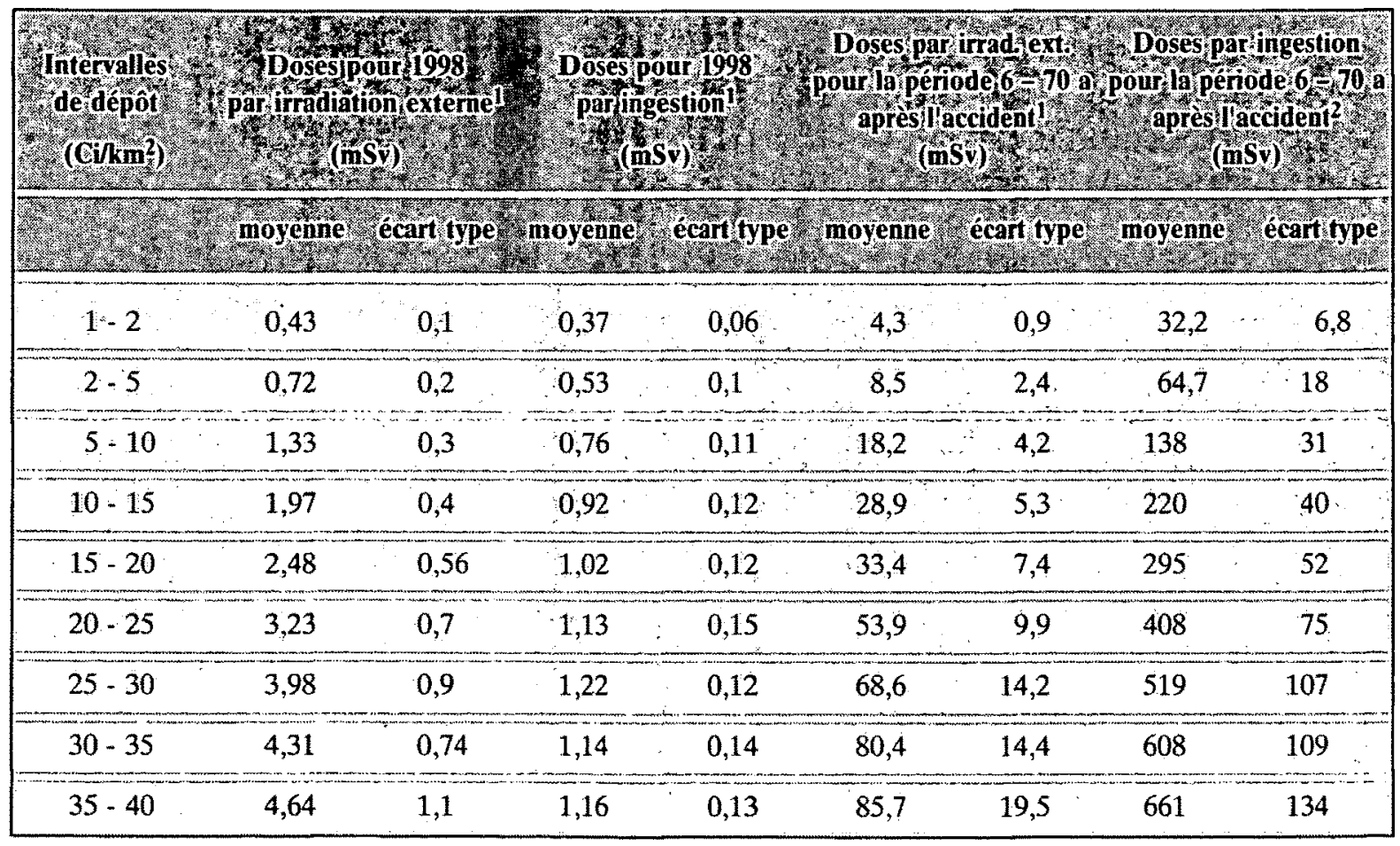

1. Avec mesures de protection et contre-mesures agricoles.

2. Sans mesures de protection et contre-mesures agricoles.

\section{Explications complêmentaires sur les valeurs mentionnées :}

Pour chaque zone de dépôt, plusieurs enregistrements (doses par irradiation ou ingestion) étaient disponibles. Les moyennes et les écart-types associés ont été calculés en supposant une répartition normale des valeurs, ce qui se vérifie de façon satisfaisante.

On constate que la relation entre les zones de dépôts et l'irradiation externe associée (colonnes 1 et 2 ) n'est pas linéaire. Cela est dû à la mise en cuvre de contre-mesures et restrictions diverses plus sévères à mesure que les dépôts augmentent, ce qui est confirmé par l'étude du rapport entre les doses avec mesures de protection (colonne 2) et les doses sans mesures de protection (colonne 6) qui sont à peu près proportionnelles aux dépôts. Malheureusement, les précisions sur ces mesures graduelles de protection sont absentes des données. 